\title{
Downregulation of survivin by siRNA inhibits invasion and promotes apoptosis in neuroblastoma SH-SY5Y cells
}

\author{
L. Zhang ${ }^{1}$, H. Liang ${ }^{1}$, W. $\mathrm{CaO}^{2}$, R. Xu ${ }^{1}$ and X.L. Ju ${ }^{1}$ \\ ${ }^{1}$ Department of Pediatrics, Qilu Hospital, Shandong University, Jinan, China \\ ${ }^{2}$ Department of Obstetrics, Qingdao Central Hospital, Qingdao, China
}

\begin{abstract}
Neuroblastoma is a solid tumor that occurs mainly in children. Malignant neuroblastomas have a poor prognosis because conventional chemotherapeutic agents are not very effective. Survivin, a member of the inhibitor of the apoptosis protein family, plays a significant role in cell division, inhibition of apoptosis, and promotion of cell proliferation and invasion. Previous studies found that survivin is highly expressed in some malignant neuroblastomas and is correlated with poor prognosis. The aim of this study was to investigate whether survivin could serve as a potential therapeutic target of human neuroblastoma. We employed RNA interference to reduce survivin expression in the human neuroblastoma SH-SY5Y cell line and analyzed the effect of RNA interference on cell proliferation and invasion in vitro and in vivo. RNA interference of survivin led to a significant decrease in invasiveness and proliferation and increased apoptosis in SH-SY5Y cells in vitro. RNA interference of survivin inhibited tumor growth in vivo by $68 \pm 13 \%(P=0.002)$ and increased the number of apoptotic cells by $9.8 \pm 1.2 \%(P=0.001)$ compared with negative small interfering RNA (siRNA) treatment controls. Moreover, RNA interference of survivin inhibited the formation of lung metastases by $92 \%(P=0.002)$ and reduced microvascular density by $60 \%(P=0.0003)$. Survivin siRNA resulted in significant downregulation of survivin mRNA and protein expression both in vitro and in vivo compared with negative siRNA treatment controls. RNA interference of survivin was found to be a potent inhibitor of SH-SY5Y tumor growth and metastasis formation. These results support further clinical development of RNA interference of survivin as a treatment of neuroblastoma and other cancer types.
\end{abstract}

Key words: Neuroblastoma; Survivin; Apoptosis; Invasion; RNA interference

\section{Introduction}

Neuroblastoma is the predominant tumor of early childhood. It is the most common extracranial solid neoplasm in children, is responsible for $7 \%$ of malignancies in patients younger than 15 years of age, and represents $\sim 15 \%$ of deaths from childhood cancer (1). Based on the International Neuroblastoma Staging System, which considers stage, group, and tumor biology, patients are assigned to low-, intermediate-, or high-risk groups (2). Despite advanced therapies including surgery, radiotherapy, and chemotherapy, children with high-risk neuroblastoma have an extremely poor prognosis (3). The 5 -year survival rate of high-risk patients is as low as $20-25 \%$, and most patients develop metastatic dissemination (4). Therefore, novel therapies are urgently needed.

Survivin is an inhibitor of apoptosis protein and is overexpressed in a wide spectrum of tumors, including neuroblastoma $(5,6)$. As a known regulator of apoptosis, survivin also has a positive relationship with lymphatic metastasis in breast cancer (7), primary laryngeal and cervical carcinoma (8), gastric cancer $(9,10)$, and prostate carcinoma (11). Therefore, the inhibition of survivin by itself, or in combination with other approaches, has emerged as a promising therapeutic strategy.

RNA interference by small interfering RNA (siRNA) is seen as a useful tool for successfully silencing genes. After uptake by cells, siRNAs are delivered to endoribonucleasecontaining complexes, known as RNA-induced silencing complexes. Within this complex, the siRNA binds to a complementary RNA molecule, and the target RNA is then cleaved and destroyed (12). Gene silencing by siRNA is effective and specific to the target gene and has many potential applications in cancer treatment (13). The aim of this study was to investigate whether survivin could be a potential therapeutic target of human neuroblastoma by RNA-induced silencing. 


\section{Material and Methods}

\section{Cell culture}

SH-SY5Y and IMR32 human neuroblastoma cell lines were obtained from the American Type Culture Collection. The SH-SY5Y cells are aggressive and poorly differentiated. Cells were cultured in a 1:1 mixture of Dulbecco's modified Eagle's medium (DMEM) and Ham's F12 medium, supplemented with $2 \mathrm{mM}$ L-glutamine and $10 \%$ heat-inactivated fetal bovine serum (FBS), at $37^{\circ} \mathrm{C}$ in a humidified $5 \% \mathrm{CO}_{2}$ atmosphere.

\section{Animals}

BALB/C nude mice (4-6 weeks of age) were used for all experiments. Animals were housed in the Experimental Animal Centre of Qilu Hospital, and procedures were approved by the Institutional Animal Care and Use Committee of Qilu Hospital.

\section{SiRNA transfection}

SH-SY5Y cells were transiently transfected with $2 \mu \mathrm{g}$ survivin siRNA or control siRNA for $48 \mathrm{~h}$ using the Lipofectamine Plus ${ }^{\mathrm{TM}}$ reagent (Gibco BRL, USA) according to the manufacturer's protocol. Survivin siRNA or control siRNA was selected with $400 \mu \mathrm{g} / \mathrm{mL}$ G418 (gentamicin) during a period of 3 weeks and used for further study.

\section{Western blotting}

Forty-eight hours after transfection with survivin SiRNA or control siRNA, attached and floating cells were harvested on ice. Cells were lysed with Laemmli buffer [20\% glycerol, 4\% sodium dodecyl sulfate (SDS), $100 \mathrm{mM}$ Tris- $\mathrm{HCl}, \mathrm{pH}$ 6.8]. Protein was quantified with the RC-DC protein assay (Bio-Rad Inc., USA). Lysates were separated on a $10 \%$ SDS-polyacrylamide gel electrophoresis gel and electroblotted onto a transfer membrane (Millipore, UK). Blocking and incubation were performed in $2.5-5 \%$ Tris-buffered saline using standard procedures. Primary antibodies used were anti-survivin rabbit polyclonal antibody and anti- $\beta$-actin mouse monoclonal antibody (Abcam, USA). The secondary antibody used was a secondary sheep anti-mouse antibody (Amersham, Belgium). Proteins were visualized using an electrochemoluminescence detection kit (Amersham).

\section{Reverse transcriptase-polymerase chain reaction (RT-PCR)}

RT-PCR experiments were conducted to monitor the knockdown of survivin mRNA expression after treatment with survivin siRNA. SH-SY5Y cells were grown at $37^{\circ} \mathrm{C}$ in $5 \% \mathrm{CO}_{2}$ and transfected with survivin siRNA or control siRNA for $48 \mathrm{~h}$. Total RNA was isolated from the cells $\left(1 \times 10^{6}\right.$ cells/sample) using TRIzol ${ }^{\circledR}$ Reagent (Invitrogen, USA). The following primer sequences for PCR amplifications of genes were used: survivin (forward: $5^{\prime}$-CATTCA
AGAACTGGCCCTTC-3' and reverse: 5'-CTAAGACATT GCTAAGGGGC-3') and GAPDH (forward: 5'-ATGGGGA AGGTGAAGGTCGG-3' and reverse: 5'-AGACGCCAGT GGACTCCACGACG-3'). Complementary DNA was synthesized using a SuperScript ${ }^{\mathrm{TM}}$ one-step RT-PCR kit on a PCR cycler by heating to $50^{\circ} \mathrm{C}$ for $30 \mathrm{~min}$, followed by 30 cycles of amplification (denaturation at $94^{\circ} \mathrm{C}$ for $15 \mathrm{~s}$, annealing at $55^{\circ} \mathrm{C}$ for $30 \mathrm{~s}$, and extension at $72^{\circ} \mathrm{C}$ for $1 \mathrm{~min}$ ), and a final extension step at $72^{\circ} \mathrm{C}$ for $10 \mathrm{~min}$. The RT-PCR products were resolved by electrophoresis on $1 \%$ agarose gels, stained with ethidium bromide $(1 \mu \mathrm{g} / \mathrm{mL})$, and visualized using a UV chamber (Alpha Innotech, USA). Expression of GAPDH was used as an internal standard.

\section{Cell proliferation assay}

SH-SY5Y cells of G418-selected transfectants were seeded on 96 -well plates at a concentration of $5 \times 10^{3}$ per well 1 day before the experiment. 3-(4,5-Dimethylthiazol2-yl)-2,5-diphenyltetrazolium bromide (MTT, $0.5 \mathrm{mg} / \mathrm{mL}$; Sigma-Aldrich, USA) was added to each well at 1, 2, 3, 4, or 5 days after seeding. Generally, cells were cultured at $37^{\circ} \mathrm{C}$ for $4 \mathrm{~h}$, and then $150 \mu \mathrm{L}$ dimethyl sulfoxide was added. The absorption was measured at a wavelength of $490 \mathrm{~nm}$.

\section{Soft agar colony formation assay}

The assay was conducted on 6-well plates. Each well contained a bottom layer of $1 \%$ agarose, a middle layer of $0.5 \%$ agarose that included $7.5 \times 10^{3}$ stably transfected SH-SY5Y cells, and a top layer of media. The media in the top layer was changed every sixth day. After 14 days, cells were stained with Giemsa solution (Gibco BRL) and counted using the Quantity One ${ }^{\mathbb{R}}$ analysis software (Bio-Rad Inc.).

\section{In vitro terminal deoxynucleotidyl transferase dUTP nick-end labeling (TUNEL) assay}

Cells were plated at a density of $1 \times 10^{4}$ cells per well on 24-well plates. A constant amount of $100 \mathrm{nM}$ siRNA was added to the cell culture in each well. After incubating the cells for $48 \mathrm{~h}$, the TUNEL assay was performed using an in situ Cell Death Detection kit (Roche, Germany) according to the manufacturer's protocol. All assays were performed in quadruplicate.

\section{Invasive assay in vitro}

BD BioCoat Matrigel ${ }^{\mathrm{TM}}$ invasion chambers including polyethylene terephthalate filters coated with Matrigel basement membrane matrix ( 6 wells, $8 \mu \mathrm{m}$ pore size; BD Biosciences, USA) were rehydrated just before the assay using FBS-free DMEM according to the manufacturer's instructions. The chambers were assembled using freshly prepared Matrigel-coated filters, and DMEM containing $0.8 \mathrm{~mL} \mathrm{NIH}-3 \mathrm{~T} 3$ was added as a chemoattractant in the lower compartment. G418-selected transfectants were harvested by trypsinization, and suspended in DMEM 
containing $10 \%$ FCS. The cells (at a concentration of $1.25 \times 10^{5}$ cells $/ 2 \mathrm{~mL}$ ) were added to the invasion chamber containing a Matrigel-coated filter, and the assembled chambers were incubated for $24 \mathrm{~h}$ at $37^{\circ} \mathrm{C}$. At the end of the incubation, nonmigrating cells, which remained on the upper surface of the filter, were completely removed by wiping with a cotton swab. The cells on the bottom surface of the filter were fixed with $100 \%$ ethanol for $30 \mathrm{~s}$ and stained with toluidine blue for $10 \mathrm{~min}$. Cells that migrated into the lower chamber were counted. Experiments were repeated in triplicate.

In vivo xenograft model of growth and metastases

For metastasis observation, immunodeficient, 4-weekold male mice were purchased from Institute of Zoology, Chinese Academy of Sciences (Shanghai, China). Autoclaved cages containing food and water were changed once per week, and mouse body weight was measured every 3-4 days. SH-SY5Y cells $\left(5 \times 10^{6}\right)$, in which survivin was silenced, were injected into the abdominal cavity of the mice. The experiments were terminated after 21 days. Animals were killed by $\mathrm{CO}_{2}$ inhalation, and autopsies were performed for the assessment of metastases using the number of seeded tumors in the abdominal cavity.

For growth observation, xenograft tumors were established by subcutaneous injection of stably transfected SH-SY5Y cells $\left(5 \times 10^{6}\right)$ into the flanks of 4-week-old female nude mice ( $n=6$ per group). The experiments were terminated after 21 days. Tumor growth was monitored three times per week by calipers, and tumor volumes were calculated according to the formula (length $\times$ width $^{2}$ )/2.

\section{Immunohistochemistry}

Tumors were harvested from nude mice at the end of the experiments on day 21. Immunohistochemical studies were done on 4- $\mu \mathrm{m}$ thick sections obtained from zinc-fixed, paraffin wax-embedded tumor tissue blocks. Sections were mounted on slides, dewaxed, and rehydrated, and endogenous peroxidase activity was quenched before survivinspecific immunohistochemical staining. After specific staining or hematoxylin and eosin staining, sections were dehydrated in alcohol, cleared in xylene, and coverslipped for microscopic observation.

\section{In vivo TUNEL detection}

Histological sections were deparaffinized, hydrated in deionized water, and rinsed with phosphate-buffered saline (PBS). The sections were treated with $20 \mu \mathrm{g} / \mathrm{mL}$ proteinase $\mathrm{K}$ for 15 min to digest protein, and with $3 \% \mathrm{H}_{2} \mathrm{O}_{2}$ for 5 min to quench endogenous peroxidase activity. After they were washed with PBS, equilibration buffer was added. The slides were then treated with $10 \mu \mathrm{L}$ working-strength terminal deoxynucleotidyl transferase enzyme at $37^{\circ} \mathrm{C}$ for $60 \mathrm{~min}$. Subsequently, the sections were incubated with preheated working-strength stop solution for $10 \mathrm{~min}$, with

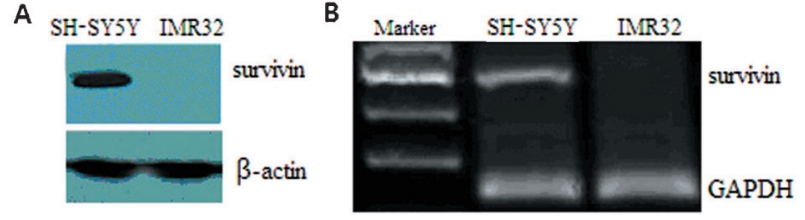

Figure 1. Expression of survivin in neuroblastoma cell lines. $A$, Representative Western blots to show the presence of survivin in neuroblastoma SH-SY5Y and IMR32 cell lines. Expression of $\beta$ actin was used as a loading control. $B$, Representative RT-PCR to show the expression of survivin mRNA. The expression of $G A P D H$ mRNA was used as a loading control.

anti-digoxigenin horseradish peroxidase (POD) for $30 \mathrm{~min}$ and with a metal-enhanced diaminobenzidine kit (Pierce, China) for 3-6 min, and washed with PBS or deionized water after each incubation. Finally, the sections were counterstained with methyl green (Vector stock solution) or Mayer's hematoxylin, and mounted on slides. The percentage of apoptotic cells was calculated as the number of apoptotic cells per number of total cells times 100 .

\section{Statistical analysis}

Data are reported as means $\pm \mathrm{SD}$. Experiments were repeated three times, with triplicate samples for each. Data were analyzed by ANOVA and the Mann-Whitney $U$-test. Statistical significance was defined as $\mathrm{P}<0.05$.

\section{Results}

\section{Overexpression of survivin in neuroblastoma cell lines}

We examined the expression of survivin in IMR32 and SH-SY5Y cell lines. Western blotting showed high expression of survivin protein in SH-SY5Y cells and very low expression in IMR32 cells (Figure 1A). Further, RT-PCR analysis showed similar variations in survivin expression in SH-SY5Y and IMR32 cells (Figure 1B). Based on the findings, we used the SH-SY5Y cell line for further experiments.

\section{Downregulation of survivin mRNA and protein levels in SH-SY5Y cells}

We examined the downregulation of survivin mRNA and protein levels in neuroblastoma $\mathrm{SH}-\mathrm{SY} 5 \mathrm{Y}$ cells after stable survivin siRNA or control siRNA transfection. RTPCR and Western blot analyses showed the extent of survivin downregulation at the protein (Figure 2A) and mRNA (Figure 2B) levels, respectively, in SH-SY5Y cells. No changes in mRNA and protein levels were observed when cells were transfected with the control siRNA. We used GAPDH mRNA expression and $\beta$-actin protein expression as internal controls for RT-PCR and Western blotting, respectively. Downregulation of survivin inhibited cell proliferation and decreased colony-forming ability.

To confirm the effect of survivin inhibition on cell 


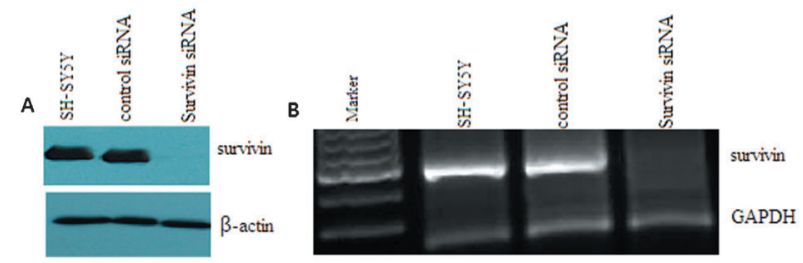

Figure 2. Alterations in survivin mRNA and protein levels in $\mathrm{SH}$ SY5Y cells after stable transfection with survivin siRNA or control siRNA. A, Western blotting for examining expression of survivin protein. Western blots were reprobed for $\beta$-actin content to demonstrate that all lanes were loaded with equal amounts of protein. $B, \mathrm{RT}-\mathrm{PCR}$ analysis for survivin mRNA expression. The expression of GAPDH mRNA was used as an internal control.

growth, cells stably transfected with survivin siRNA were cultured. The MTT assay showed that the survivin siRNAtransfected SH-SY5Y cells grew more slowly than control siRNA-transfected cells (Figure $3 \mathrm{~A} ; \mathrm{P}<0.05$ ). We used a soft agar colony formation assay to explore the effect of survivin knockdown on tumorigenesis in vitro, and the results showed that colony formation by survivin siRNAtransfected cells was decreased compared with that of control siRNA-transfected cells (Figure 3B; $\mathrm{P}<0.05$ ).

\section{Effects of survivin siRNA on cell apoptosis in vitro}

To further verify the effects of survivin siRNA, TUNEL analysis was conducted to quantify cell apoptosis. The numbers of TUNEL-positive cells were counted to obtain the percentage of apoptotic cells. As shown in Figure 4, survivin SiRNA transfection (day 2) resulted in the highest
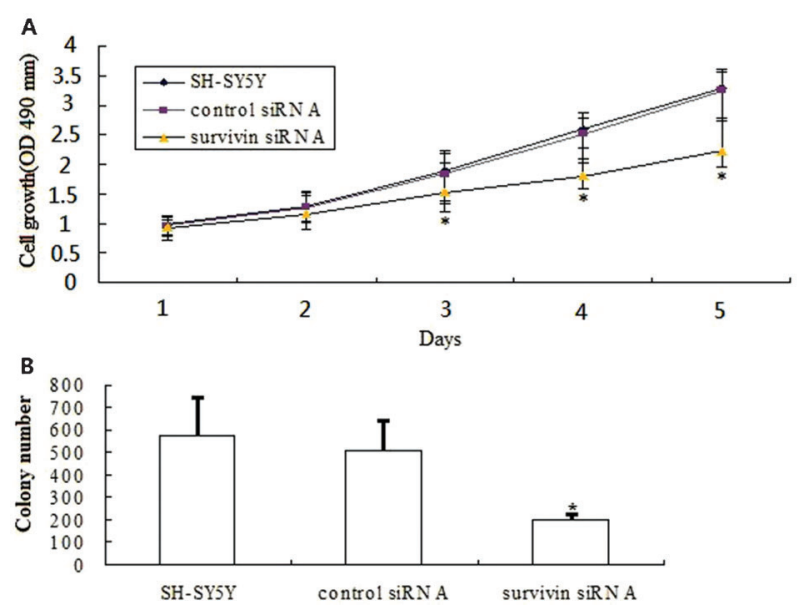

Figure 3. Influence of survivin knockdown on cell growth and colony number. A, MTT assay was analyzed for SH-SY5Y cells left untreated, transfected with survivin siRNA, and transfected with control siRNA on days $1,2,3,4$, and 5 . B, Soft agar assay was assessed to evaluate the colony-forming ability of the cells. Data are reported as means $\pm S D$. ${ }^{*} \mathrm{P}<0.05$ compared with untreated SH-SY5Y or control siRNA-transfected cells (MannWhitney U-test).

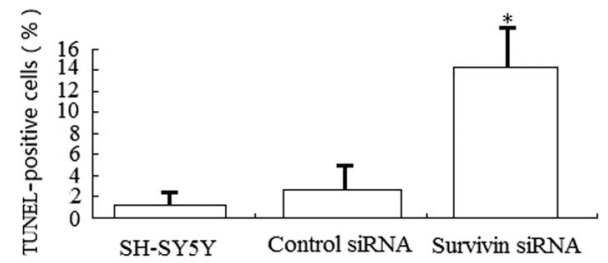

Figure 4. Influence of survivin knockdown on cell apoptosis. TUNEL staining was used to detect apoptosis in SH-SY5Y cells after transient survivin siRNA and control siRNA transfection for $48 \mathrm{~h}$. Data are reported as means $\pm S D$. ${ }^{*} \mathrm{P}<0.05$, compared with untreated SH-SY5Y cells or control siRNA-transfected cells (Mann-Whitney U-test).

level of cell apoptosis ( $14.3 \pm 2.7 \%)$, which was significantly higher than the level induced by transfection with the control $\operatorname{siRNA}(2.8 \pm 0.8 \%)$.

\section{Downregulation of survivin inhibits cell migration in SH-SY5Y cells in vitro}

We examined the effects of survivin knockdown on the ability of SH-SY5Y cells to migrate through a Matrigelcoated membrane. Cells below the membrane that migrated through the Matrigel coating were stained and examined by light microscopy. We found that control siRNA transfection did not prevent cells from migrating through the Matrigel-coated membrane, but survivin SiRNA transfection significantly reduced cell migration (Figure 5).

\section{In vivo inhibition of tumor growth and metastasis}

Two deaths were noted in the survivin siRNA and control siRNA groups before the end of the 21-day treatment period because of large tumors. Conversely, all mice receiving survivin siRNA survived and exhibited a healthier appearance. As shown in Figure 6A, tumor volumes were significantly smaller in the survivin siRNA group $(68 \pm 13 \%, P=0.002)$ relative to the control siRNA group. Control siRNA failed to inhibit tumor growth.

On the basis of the effects of survivin siRNA on tumor growth, we then tested the potential for survivin siRNA to enhance apoptosis in vivo. As shown in Figure 6B, significant increases in apoptosis were not seen in the control siRNA groups. However, apoptosis was significantly

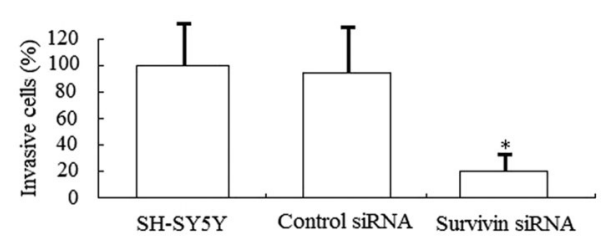

Figure 5. Cell migration assay in neuroblastoma SH-SY5Y cells. Quantitation of Matrigel-invaded cells underneath the membrane. Data are reported as means \pm SD of 3 independent experiments. ${ }^{*} \mathrm{P}<0.01$, compared with control siRNA (Mann-Whitney U-test). 


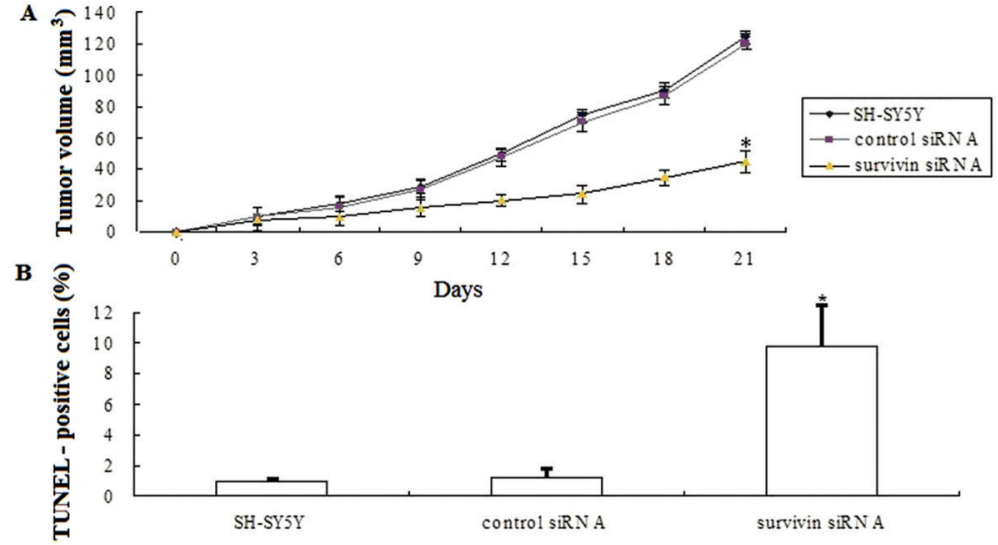

C

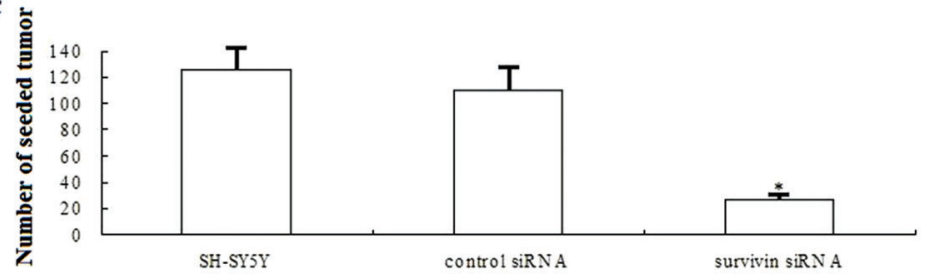

Figure 6. Tumor growth and metastasis inhibition in vivo by survivin siRNA. A, Survival curves of pseudometastatic neuroblastoma-bearing mice in response to survivin small interfering RNA (survivin siRNA) and control siRNA $(n=8)$. $B$, Terminal deoxynucleotidyl transferase-mediated nick-end labeling (TUNEL) staining performed on formalin-fixed and paraffin-embedded tumor sections of SH-SY5Y subcutaneous tumors. TUNEL staining revealed a massive induction of apoptosis by survivin siRNA. $C$, Seeded tumors in the abdominal cavity organs of each animal were evaluated for the extent of metastatic invasion. Data are reported as means \pm SD of 3 independent experiments. ${ }^{*} \mathrm{P}<0.05$, compared to control siRNA (Mann-Whitney U-test). increased by $9.8 \pm 1.2 \%(P=0.01)$ in tumors treated with survivin siRNA.

Immunohistochemical staining revealed high levels of survivin expression in the orthotopic tumors in the control siRNA group and low levels of survivin expression in the orthotopic tumors of the survivin siRNA group (data not shown).

We then tested the effect of survivin siRNA on $\mathrm{SH}$ SY5Y pseudometastatic models. The mice were injected with $\left(5 \times 10^{6}\right) \mathrm{SH}-\mathrm{SY} 5 \mathrm{Y}$ cells in which survivin was silenced (18 animals) or control siRNA-transfected (18 animals) SHSY5Y cells into the abdominal cavity on day 0 . After 21 days, the animals were killed, and autopsies were performed to remove organs. There were many off-white nodules in the peritoneum, retina, mesentery, intestinal, and gastric walls. Autopsy results revealed a reduction of seeded tumor nodules in the abdominal organs in the survivin siRNA-transfected group. The number of seeded tumors was $126.8 \pm 12.6,110.7 \pm 14.4$, and $26.87 \pm 8.4$ in the SH-SY5Y, control siRNA-transfected, and survivin siRNA-transfected groups, respectively. Differences in the number of seeded tumors reached statistical significance $(P=0.013$; Figure $6 C)$. Immunohistochemical staining revealed high levels of survivin expression in the control siRNA group and low levels in the survivin siRNA group (data not shown).

\section{Discussion}

Despite many advances in the past three decades, neuroblastoma has remained an enigmatic challenge to clinical and basic scientists. A large body of data from basic research into genes and oncogenes has accumulated. Increased or decreased expression of the MYCN, H-ras, and $\operatorname{trk} A$ genes in neuroblastoma is well known (14-17). However, the continuing poor prognosis for advanced neuroblastoma reflects, in part, a lack of knowledge of the tumor's basic biology.

Previous data have demonstrated that survivin expression is increased in human neuroblastoma tissues and cultured cell lines $(5,6,18)$. The expression level of survivin has been correlated with the clinical staging of neuroblastoma. Multivariate analysis has suggested that survivin may be an independent prognostic biomarker for neuroblastoma. In our current study, we evaluated the possibility of survivin as a therapeutic target of neuroblastoma.

In the present study, we observed that survivin was overexpressed in human malignant neuroblastoma $\mathrm{SH}$ SY5Y cells, and very slightly expressed in the IMR32 cell line. Thus, survivin could be a potential therapeutic target for the treatment of malignant neuroblastoma SH-SY5Y cells.

Recently, the problem of "off-target" effects from RNA interference has been documented (19). In studies not presented here, we established a distinct line of survivinsuppressed SH-SY5Y cells in which gene expression analysis confirmed that survivin was downregulated (20). In the present study, we successfully employed survivin siRNA to knock down survivin mRNA and protein levels in human malignant neuroblastoma SH-SY5Y cell lines. We found that siRNA directed against survivin resulted in complete suppression of survivin expression at the mRNA and protein levels in SH-SY5Y cells. In this study, we found that, after transfection with survivin siRNA, the survivin 
mRNA level and protein expression decreased; meanwhile cell growth was inhibited and apoptosis increased in vitro. Therefore, our data confirmed that survivin serves to regulate both cell proliferation and survival. We observed that targeted disruption of survivin in SH-SY5Y cells resulted in a significant decrease in cell invasion across an artificial matrix that mimics an in vivo extracellular matrix. Therefore, our data also confirmed that survivin serves to regulate invasive ability.

Because survivin plays an important role in the growth and invasion of neuroblastoma cells in vitro, we then explored the role of survivin in vivo. Our study showed that survivin siRNA-transfected tumors grew very slowly,

\section{References}

1. Maris JM, Hogarty MD, Bagatell R, Cohn SL. Neuroblastoma. Lancet 2007; 369: 2106-2120, doi: 10.1016/S01406736(07)60983-0.

2. Haase GM, Perez C, Atkinson JB. Current aspects of biology, risk assessment, and treatment of neuroblastoma. Semin Surg Oncol 1999; 16: 91-104, doi: 10.1002/(SICl)10982388(199903)16:2<91::AID-SSU3>3.0.CO;2-1.

3. Laverdiere C, Cheung NK, Kushner BH, Kramer K, Modak $\mathrm{S}$, LaQuaglia MP, et al. Long-term complications in survivors of advanced stage neuroblastoma. Pediatr Blood Cancer 2005; 45: 324-332, doi: 10.1002/pbc.20331.

4. Tonini GP, Pistoia V. Molecularly guided therapy of neuroblastoma: a review of different approaches. Curr Pharm Des 2006; 12: 2303-2317, doi: 10.2174/138161206777585193.

5. Ito R, Asami S, Motohashi S, Ootsuka S, Yamaguchi $Y$, Chin M, et al. Significance of survivin mRNA expression in prognosis of neuroblastoma. Biol Pharm Bull 2005; 28: 565568, doi: $10.1248 / \mathrm{bpb} .28 .565$.

6. Azuhata T, Scott D, Takamizawa S, Wen J, Davidoff A, Fukuzawa $\mathrm{M}$, et al. The inhibitor of apoptosis protein survivin is associated with high-risk behavior of neuroblastoma. J Pediatr Surg 2001; 36: 1785-1791, doi: 10.1053/jpsu.2001.28839.

7. Cai X, Ma S, Gu M, Zu C, Qu W, Zheng X. Survivin regulates the expression of VEGF-C in lymphatic metastasis of breast cancer. Diagn Pathol 2012; 7: 52, doi: 10.1186/1746-15967-52.

8. Marioni G, Bertolin A, Giacomelli L, Marchese-Ragona R, Savastano M, Calgaro N, et al. Expression of the apoptosis inhibitor protein Survivin in primary laryngeal carcinoma and cervical lymph node metastasis. Anticancer Res 2006; 26: 3813-3817.

9. Lee GH, Joo YE, Koh YS, Chung IJ, Park YK, Lee JH, et al. Expression of survivin in gastric cancer and its relationship with tumor angiogenesis. Eur J Gastroenterol Hepatol 2006; 18: 957-963, doi: 10.1097/01.meg.0000230086.83792.56.

10. Miyachi K, Sasaki K, Onodera S, Taguchi T, Nagamachi M, Kaneko $\mathrm{H}$, et al. Correlation between survivin mRNA expression and lymph node metastasis in gastric cancer. Gastric Cancer 2003; 6: 217-224, doi: 10.1007/s10120-003-0255-2.

11. Shariat SF, Lotan $Y$, Saboorian H, Khoddami SM, and there were many more apoptitic cells in the survivin siRNA-transfected tumors compared with the controls. We tested the effect of survivin siRNA transfection in SH-SY5Y pseudometastatic models. The results showed that fewer metastatic nodules were found in the survivin siRNAtransfected than in the control groups. In this study, we demonstrated that survivin siRNA transfection resulted in remarkable decreases in the expression of survivin in $\mathrm{SH}$ SY5Y cells. We, therefore, suggest that knockdown of survivin could inhibit metastasis and growth, and may induce apoptosis in neuroblastoma in vivo. The results of our present study suggest that knockdown of survivin could be a new adjuvant therapy for neuroblastoma.

Roehrborn CG, Slawin KM, et al. Survivin expression is associated with features of biologically aggressive prostate carcinoma. Cancer 2004; 100: 751-757, doi: 10.1002/ cncr.20039.

12. Elbashir SM, Harborth J, Lendeckel W, Yalcin A, Weber K, Tuschl T. Duplexes of 21-nucleotide RNAs mediate RNA interference in cultured mammalian cells. Nature 2001; 411: 494-498, doi: 10.1038/35078107.

13. Devi GR. siRNA-based approaches in cancer therapy. Cancer Gene Ther 2006; 13: 819-829, doi: 10.1038/sj.cgt.7700931.

14. Castleberry RP. Predicting outcome in neuroblastoma. $N$ Engl J Med 1999; 340: 1992-1993, doi: 10.1056/ NEJM199906243402510.

15. Castel V, Garcia-Miguel P, Canete A, Melero C, Navajas A, Ruiz-Jimenez JI, et al. Prospective evaluation of the International Neuroblastoma Staging System (INSS) and the International Neuroblastoma Response Criteria (INRC) in a multicentre setting. Eur J Cancer 1999; 35: 606-611, doi: 10.1016/S0959-8049(98)00395-5.

16. Castleberry RP, Pritchard J, Ambros P, Berthold F, Brodeur GM, Castel V, et al. The International Neuroblastoma Risk Groups (INRG): a preliminary report. Eur J Cancer 1997; 33: 2113-2116, doi: 10.1016/S0959-8049(97)00202-5.

17. Shimada H, Ambros IM, Dehner LP, Hata J, Joshi VV, Roald $B$, et al. The International Neuroblastoma Pathology Classification (the Shimada system). Cancer 1999; 86: 364372, doi: 10.1002/(SICI)1097-0142(19990715)86:2<364::AIDCNCR21>3.0.CO;2-7.

18. Hossain MM, Banik NL, Ray SK. Survivin knockdown increased anti-cancer effects of (-)-epigallocatechin-3-gallate in human malignant neuroblastoma SK-N-BE2 and SH-SY5Y cells. Exp Cell Res 2012; 318: 1597-1610, doi: 10.1016/j.yexcr.2012.03.033.

19. Couzin J. Molecular biology. RNAi shows cracks in its armor. Science 2004; 306: 1124-1125, doi: 10.1126/science.306. 5699.1124

20. Liang $H$, Zhang $L, X u R$, Ju XL. Silencing of survivin using YM155 induces apoptosis and chemosensitization in neuroblastomas cells. Eur Rev Med Pharmacol Sci 2013; 17: 2909-2915. 